\title{
On the Statistical Properties of Nakagami-Hoyt Vehicle-to-Vehicle Fading Channel under Nonisotropic Scattering
}

\author{
Muhammad Imran Akram and Asrar U. H. Sheikh \\ Department of Electrical Engineering, King Fahd University of Petroleum and Minerals, Dhahran 31261, Saudi Arabia \\ Correspondence should be addressed to Muhammad Imran Akram, miakram@kfupm.edu.sa
}

Received 18 March 2012; Revised 14 July 2012; Accepted 19 September 2012

Academic Editor: Ai Bo

Copyright ( $) 2012$ M. I. Akram and A. U. H. Sheikh. This is an open access article distributed under the Creative Commons Attribution License, which permits unrestricted use, distribution, and reproduction in any medium, provided the original work is properly cited.

\begin{abstract}
This paper presents the statistical properties of the vehicle-to-vehicle Nakagami-Hoyt (Nakagami-q) channel model under nonisotropic condition. The spatial time correlation function (STCF), the power spectral density (PSD), squared time autocorrelation function (SQCF), level crossing rate (LCR), and the average duration of Fade (ADF) of the Nakagami-Hoyt channel have been derived under the assumption that both the transmitter and receiver are nonstationary having nonomnidirectional antennas. A simulator that uses the inverse-fast-fourier-transform- (IFFT-) based computation method is designed for this model. The simulator and analytical results are compared.
\end{abstract}

\section{Introduction}

With passage of time, applications of wireless communication, their usefulness, and reliability are increasing. The recent applications where wireless communications is extensively used include wireless local area network (WLAN), multimedia messaging cellular telephone systems, satellite systems, femtocells, Bluetooth, and Zigbee devices. The wireless devices are also extensively used in in-car security systems equipment, home television systems private mobile systems, and so forth. In the conventional wireless communication systems, all the mobile stations communicate with each other via fixed base stations which are normally placed at elevated locations. Since mobile station is likely to be surrounded by objects having different shapes and orientations, the direct propagation path may not always present, and communication results from scattering that occurs near the mobile station. The channel between the transmitter and receiver is usually multipath fading channel; the signal fading occurs due to terminal mobility.

An in-depth knowledge leads to an accurate model of mobile propagation channel which is essential for a simulator design that provides dependable performance results. Over the past many years, several mobile channel models have been proposed for links between fixed base station and mobile station. These include short-term fading models like the well-known Rayleigh, Rice [1], Hoyt [2], Nakagami-m [3], and Weibull [4]. For long-term fading model, lognormal distribution has been used $[5,6]$. Several composite fading models combining the effects of short- and long-term fading (Nakagami-lognormal [3], Suzuki [7], and Rice-lognormal [8]) have also been proposed.

Over the past decade, the research has been focused on vehicle-to-vehicle (V2V) or mobile-to-mobile (M2M) communication systems where no base station is present and both the transmitter $T x$ and the receiver $R x$ are in motion. The V2V communication finds applications in mobile adhoc wireless networks, intelligent highway systems, emergency, military, and security vehicles. The antennas are mounted on the top or on the side of vehicles, which move with different velocities and resulting in time varying channels. The buildings and other obstacles surrounding the terminals act as scatterers thereby generating multipaths. Depending upon the vehicular positions, the line of sight (LOS) may or may not be present.

During the past decade, a large number of research projects have been done on V2V communications [9-12]. Reference [13] presents a survey of the vehicular channel characterization under different environments (highway, 
rural, urban, and suburban). It shows the path loss exponent, Root Mean Square (RMS) delay spread, and mean Doppler spread under these conditions. The statistical model for vehicle-to-vehicle communication was first proposed by Akki and Haber [14], and its statistical properties were reported in [15]. Based on the work of [15], many V2V simulators were designed and implemented. Reference [16] presented a discrete line spectrum-based approach to simulate the channel. The work reported in [17] is based on sum of sinusoids (SOS) approach for simulator design. In [18], the simulation of multiple input multiple output (MIMO) V2V is presented. The simulator proposed in [19] is based on Kullback-Leibler divergence which is compared with IFFT based approach of simulator design. Reference [20] uses Gaussian quadrature rule for simulator design. Reference [21] proposes an efficient SOS-based approach for $\mathrm{V} 2 \mathrm{~V}$ simulator design. All the simulator design approaches mentioned above were designed for V2V Rayleigh fading channels.

A small number of works have used non-Rayleigh fading channel models. The second order statistics of NakagamiHoyt channel have been derived in [22]. Reference [23] derives the statistical properties of double Nakagami-Hoyt channel.

In many real world scenarios, nonisotropic scattering is often experienced by both the mobile transmitter and receiver. It has been shown in [24-26] that in dense urban environments, non-isotropic scattering around the mobile station exists. Reference [27] derives the second order statistics of V2V Ricean fading channel under non-isotropic conditions and compares the theoretical results with the measured data. Reference [28] derives the autocorrelation function of Rice process under non-isotropic condition. Reference [29] presented V2V model for Rayleigh fading under non-isotropic condition. Many nonuniform distributions have been discussed for angle of arrival (AOA) and angle of departure (AOD). These include Gaussian, Laplacian, quadratic, and Von Misses distributions. Von Misses distribution (assumed in this paper), a generic case described in [24], covers the other distributions (Gaussian, Laplacian, cosine, and uniform distributions) as its special cases.

In this paper, a novel V2V Nakagami-Hoyt channel model under non-isotropic scattering condition is proposed. The existing channel models $[2,15,30]$ are treated as its special cases. Analytical expressions for second order statistical properties including STCF, PSD, SQCF, LCR, and $\mathrm{ADF}$ of the envelop of the proposed model have also been derived. An IFFT based simulator has been developed to validate the first and second order statistical parameters of the proposed model. Mean Square Error (MSE) of autocorrelation function is also plotted to show the simulator accuracy. To the best of authors' knowledge, no work has been reported on the statistical parameters of the proposed model and its simulation.

The remainder of this paper is organized as follows. Section 2 presents details of the Nakagami-Hoyt V2V channel model and its first and second order statistics. Section 3 describes the simulation method, results of the simulator, and their comparison with the analytical results. Finally, Section 4 concludes the paper.

\section{The Nakagami-Hoyt Channel Model}

The Nakagami-Hoyt (also known as q) distribution is the distribution of the modulus of a complex Gaussian random variable whose components are uncorrelated with zero mean and unequal variances.

In this section, we briefly describe the proposed channel model along with its usefulness and derive the first and second order statistics of Hoyt fading channel under the assumptions that the channel is narrow band, and the receiver and transmitter are moving with velocities $V_{1}$ and $V_{2}$, respectively and the non-isotropic scattering (i.e, AOA and AOD have nonuniform distributions).

A Hoyt process, $R(t)$, is obtained by complex Gaussian random process as

$$
\begin{gathered}
\mu(t)=\mu_{1}(t)+j \mu_{2}(t), \\
R(t)=|\mu(t)|,
\end{gathered}
$$

where $j=\sqrt{-1}, \mu_{1}(t)$ and $\mu_{2}(t)$ are complex Gaussian random processes with zero means and variances $\sigma_{1}^{2}$ and $\sigma_{2}^{2}$, respectively. $|\cdot|$ indicates the $L_{2}$ norm.

The parameters $q$ and $a$ are defined as

$$
\begin{aligned}
& q=\frac{\sigma_{1}}{\sigma_{2}}, \\
& a=\frac{V_{2}}{V_{1}} .
\end{aligned}
$$

Reference [14] proposed a statistical model for the mobile-to-mobile Rayleigh fading channel. This model is modified for the Nakagami-Hoyt frequency flat fading channel. The baseband equivalent channel impulse response is given as

$$
\mu_{1}(t)=\sum_{n=1}^{N} r_{n} \cos \left(\left(w_{1 n}+w_{2 n}\right) t+\phi_{n}\right) .
$$

Also

$$
\mu_{2}(t)=\frac{1}{q} \sum_{n=1}^{N} r_{n} \sin \left(\left(w_{1 n}+w_{2 n}\right) t+\phi_{n}\right),
$$

where $N$ is the number of propagation paths; $r_{n}$ and $\phi_{n}$ are the uniformly distributed amplitude and phase of $n$th path, respectively. $w_{\text {in }}$ is given by

$$
w_{\text {in }}=2 \pi f_{d i} \cos \left(\alpha_{\text {in }}\right), \quad i=1,2,
$$

where $f_{d 1}$ and $f_{d 2}$ are the maximum Doppler frequency due to the motion of receiver and transmitter, respectively. $\alpha_{1 n}$ and $\alpha_{2 n}$ are the AOA and AOD of the $n$th path with respect to the velocity vector of receiver and transmitter, respectively.

Assuming that $\alpha_{1 n}, \alpha_{2 n}$, and $\phi_{n}$ are independent for all $n$ with $\alpha_{1 n}$ and $\alpha_{2 n}$ are non-uniformly distributed having Von Mises PDF described in [24]. The PDF of the Von Mises distribution is given by

$$
p_{\alpha}(\alpha)=\frac{\exp [\kappa \cos (\alpha-\nu)]}{2 \pi I_{0}(\kappa)}, \quad \kappa>0,
$$




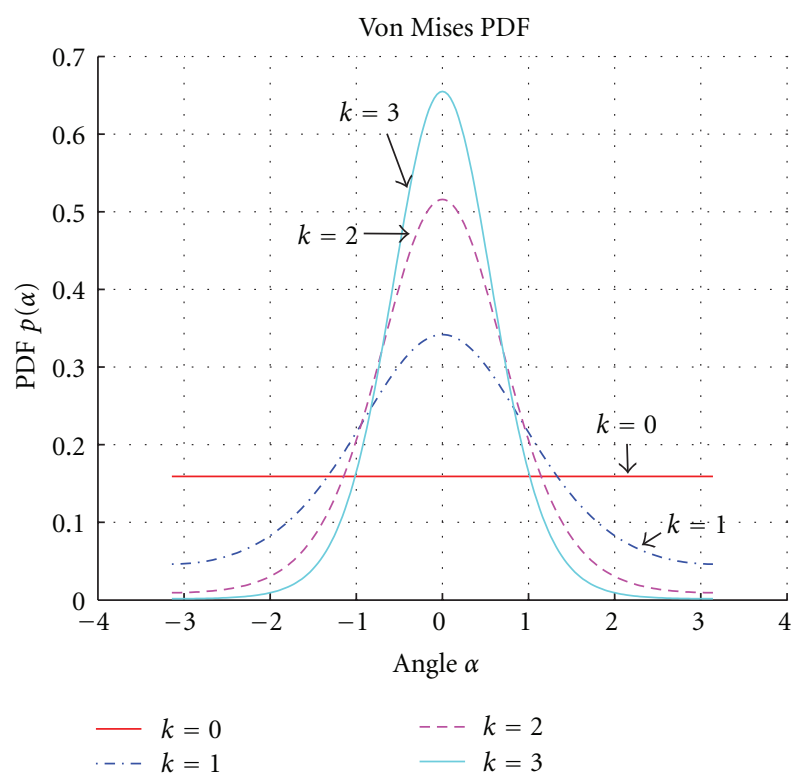

FIgURe 1: Von Mises PDF showing nonisotropic scattering.

where $I_{0}(\cdot)$ is the zero-order modified Bessel function of the first kind, $v$ is the mean direction of the AOD or AOA, and $\kappa$ is the concentration parameter which controls the width of the scatterers. Figure 1 illustrates the PDF $p_{\alpha}(\alpha)$ with different values of $\kappa$ for $v=0$. If $\kappa=0$, then the von Mises $\mathrm{PDF}$ reduces to a uniform distribution (isotropic scattering).

2.1. Usefulness of the Proposed Channel Model. The measurements made in the rural environment demonstrated that the channel is more accurately modeled only when the variance of the in-phase and the quadrature components are not identical [22]. This observation was further supported in [31] where the model matches the measured data for the cases of unequal variances. For V2V communication, reference [32] observed that when the distance between the vehicles exceeds $70 \mathrm{~m}$, the Nakagami m-factor is observed to be less than unity, which corresponds to the case of unequal variances of the component of complex Gaussian process. Further as found from the V2V measurements [33, 34], the $m$ value of each tap of the channel model described is found to be less than unity $(0.75-0.89)$ which from [22] corresponds to the value of $q(0.5-0.707)$.

The channel models described previously do not consider the following two scenarios simultaneously. First, when the antennas are not omnidirectional, and scatterers around the receiver and transmitter are not uniformly distributed. This is normally the case in V2V communications when transmit and receive antennas are present inside the vehicles. Second, when the fading is severe and the channel gains are no longer Rayleigh ie $q<1$. The proposed model is a generalized model. IT is applicable to these cases for $\mathrm{V} 2 \mathrm{~V}$ when $a \neq 0$ and base to vehicle when $a=0$ and covers the previously existing models as its special cases.

2.2. First Order Statistics. The probability density function of the envelope $R(t)$ is given by [2]

$$
\begin{aligned}
p_{R}(x)= & \frac{x}{\sigma_{1} \sigma_{2}} \exp \left[-\frac{x^{2}}{4}\left(\frac{1}{\sigma_{1}^{2}}+\frac{1}{\sigma_{2}^{2}}\right)\right] \\
& \times I_{0}\left[\frac{x^{2}}{4}\left(\frac{1}{\sigma_{1}^{2}}-\frac{1}{\sigma_{2}^{2}}\right)\right], \quad x \geq 0,
\end{aligned}
$$

where $I_{0}(\cdot)$ denotes the zeroth-order modified Bessel function of the first kind. The pdf of the corresponding phase process $\nu(t)=\arctan \left[\mu_{2}(t) / \mu_{1}(t)\right]$ is given by

$$
p_{\nu}(\theta)=\frac{\sigma_{1} \sigma_{2}}{2 \pi\left(\sigma_{2}^{2} \cos ^{2} \theta+\sigma_{1}^{2} \sin ^{2} \theta\right)}, \quad 0 \leq \theta<2 \pi
$$

Since the pdfs are independent of time so they will remain independent for V2V Nakagami-Hoyt channels. The mean $m_{\mu}$ and RMS $R_{\text {rms }}$ values can be easily obtained as

$$
\begin{aligned}
m_{\mu}=E[\mu(t)] & =E\left[\mu_{1}(t)\right]+j E\left[\mu_{2}(t)\right] \\
& =0 \\
R_{\mathrm{rms}}=\sqrt{E\left[\mu(t)^{2}\right]} & =\sqrt{E\left[\mu_{1}(t)^{2}\right]+E\left[\mu_{2}(t)^{2}\right]} \\
& =\sqrt{\sigma_{1}^{2}+\sigma_{2}^{2}} \\
& =\sigma_{2} \sqrt{1+q^{2}} .
\end{aligned}
$$

2.3. Second Order Statistics. In this section, The STCF, PSD, SQCF, LCR and ADF of the Nakagami-Hoyt V2V fading process are derived. These quantities are useful in estimation of burst error, mobile velocity and Markov modeling of fading channels [35-37].

2.3.1. Spatial Time Correlation Function. For derivation of spatial time correlation function of Nakagami-Hoyt V2V channel, [14] is used as a reference, which describes the case for Rayleigh distribution. The results are obtained for more general case where $\sigma_{1} \neq \sigma_{2}$.

The spatial time correlation function of the envelope is given by [38]

$$
\mathbf{R}\left(x_{1}, x_{2}, t_{1}, t_{2}\right)=\frac{1}{2}\left\langle\mu_{x_{2}}\left(t_{2}\right) \mu_{x_{1}}^{*}\left(t_{1}\right)\right\rangle,
$$

where $\langle\cdot\rangle$ is the statistical average $\mu_{x_{1}}\left(t_{1}\right)$ and $\mu_{x_{2}}\left(t_{2}\right)$ are the complex envelop received at positions $x_{1}$ and $x_{2}$ at time $t_{1}$ and $t_{2}$, respectively as given in [14]. It is shown in Appendix A that the general form of the spatial time correlation function is given by

$$
\mathbf{R}(\Delta x, \Delta t)=\sum_{n=0}^{1} \frac{(1+q \cos (n \pi))^{2}}{4 q^{2}} \sigma_{1}^{2} \prod_{i=1}^{2} \frac{I_{0}\left(\sqrt{\kappa_{i}^{2}-K^{2} M_{i}^{2}+j 2 \kappa_{i} K M_{i} \cos \nu_{i} \cos (n \pi)}\right)}{I_{0}\left(\kappa_{i}\right)}
$$


where

$$
M_{i}(\Delta x, \Delta t)=V_{i} \Delta t+(2-i) \Delta x
$$

$K=2 \pi / \lambda, \Delta t=t_{2}-t_{1}$ and $\Delta x=x_{2}-x_{1}$.
$\mathbf{R}(\Delta x, \Delta t)$ is the correlation functions of two signal envelopes obtained at two locations $\Delta x$ apart and at two time instant $\Delta t$ apart.

The time correlation function is obtained by setting $\Delta x=$ 0 in (11)

$$
\mathbf{R}_{\mu}(\Delta t)=\mathbf{R}(0, \Delta t)=\sum_{n=0}^{1} \frac{(1+q \cos (n \pi))^{2}}{4 q^{2}} \sigma_{1}^{2} \prod_{i=1}^{2} \frac{I_{0}\left(\sqrt{\kappa_{i}^{2}-K^{2}\left(V_{i} \Delta t\right)^{2}+j 2 \kappa_{i} K V_{i} \Delta t \cos \nu_{i} \cos (n \pi)}\right)}{I_{0}\left(\kappa_{i}\right)} .
$$

For a more specific case (isotropic scattering), substituting $\kappa_{i}=0$ and $v_{i}=0$ in (11) and simplifying,

$$
\mathbf{R}(\Delta x, \Delta t)=\frac{1+q^{2}}{2 q^{2}} \sigma_{1}^{2} J_{0}\left(K V_{2} \Delta t\right) J_{0}\left(K V_{1} \Delta t+K \Delta x\right)
$$

$J_{0}(\cdot)$ is the zero-order Bessel function. It can be shown that for $q=1$, the space time correlation function for vehicle to vehicle Rayleigh fading channel is obtained.

$$
\mathbf{R}(\Delta x, \Delta t)=\sigma_{1}^{2} J_{0}\left(K V_{2} \Delta t\right) J_{0}\left(K V_{1} \Delta t+K \Delta x\right)
$$

which matches with the result of [14]. Further by setting $V_{2}=0$ (i.e., transmitter stationary) in (15), we get $T x$ (stationary) and $R x$ (mobile) Rayleigh channel

$$
\mathbf{R}(\Delta x, \Delta t)=\sigma_{1}^{2} J_{0}\left(K V_{1} \Delta t+K \Delta x\right) .
$$

The time correlation is obtained by setting $\Delta x=0$ in (15) as

$$
\mathbf{R}_{\mu}(\Delta t)=\mathbf{R}(0, \Delta t)=\frac{1+q^{2}}{2 q^{2}} \sigma_{1}^{2} J_{0}\left(K V_{2} \Delta t\right) J_{0}\left(K V_{1} \Delta t\right) .
$$

The spatial correlation function is obtained by setting $\Delta t=0$ in (15) as

$$
\mathbf{R}_{\mu}(\Delta x)=\mathbf{R}(\Delta x, 0)=\frac{1+q^{2}}{2 q^{2}} \sigma_{1}^{2} J_{0}(K \Delta x) .
$$

Similarly, for Rayleigh fading, the time and spatial correlation functions are obtained as

$$
\begin{gathered}
\mathbf{R}_{\mu}(\Delta t)=\sigma_{1}^{2} J_{0}\left(K V_{2} \Delta t\right) J_{0}\left(K V_{1} \Delta t\right), \\
\mathbf{R}_{\mu}(\Delta x)=\sigma_{1}^{2} J_{0}(K \Delta x) .
\end{gathered}
$$

2.3.2. Power Spectral Density. The power spectral density $S(f)$ of the wide sense stationary (WSS) process is obtained by taking the Fourier transform of the time autocorrelation function as

$$
S(f)=\int_{-\infty}^{\infty} \mathbf{R}_{\mu}(\Delta t) e^{-j 2 \pi f \Delta t} d \Delta t .
$$

For non-isotropic scattering $\left(\kappa_{i} \neq 0, v_{i} \neq 0\right)$, the integral is evaluated using the Fourier transform of (13).
For isotropic scattering, the power spectral density is obtained by taking the Fourier transform of (17) as

$$
S(f)=\frac{1+q^{2}}{2 q^{2}} \sigma_{1}^{2} \int_{-\infty}^{\infty} J_{0}\left(K V_{2} \Delta t\right) J_{0}\left(K V_{1} \Delta t\right) e^{-j 2 \pi f \Delta t} d \Delta t .
$$

The integral can be evaluated using [39]. From [14], the reduced form is

$$
\begin{aligned}
S(f)= & \frac{1+q^{2}}{2 q^{2} \pi^{2} f_{m 1 \sqrt{a}}} \sigma_{1}^{2} \\
& \times K\left[\frac{(1+a)}{2 \sqrt{a}} \sqrt{1-\left(\frac{f}{(1+a) f_{m 1}}\right)^{2}}\right],
\end{aligned}
$$

where $K(\cdot)$ is the elliptical integral function of first kind; $f_{m 1}$, $f_{m 2}$ are the maximum Doppler shifts due to the motion of the receiver and transmitter, respectively with $f_{m i}=V_{i} / \lambda$. Therefore, $f_{m 2}=a f_{m 1}$.

Now, for the case $a=0$, we have $V_{2}=0$. Hence, the PSD is obtained as

$$
S(f)=\frac{1+q^{2}}{2 q^{2}} \sigma_{1}^{2} \int_{-\infty}^{\infty} J_{0}\left(K V_{1} \Delta t\right) e^{-j 2 \pi f \Delta t} d \Delta t
$$

which is evaluated in [39] as

$$
S(f)=\frac{1+q^{2}}{2 q^{2}} \frac{\sigma_{1}^{2}}{\pi \sqrt{f_{m 1}^{2}-f^{2}}}
$$

which is the expression for PSD of base to mobile Hoyt channel. Again the Rayleigh base to mobile PSD expression is obtained by setting $q=1$ in (23) as

$$
S(f)=\frac{\sigma_{1}^{2}}{\pi \sqrt{f_{m 1}^{2}-f^{2}}} .
$$

2.3.3. Squared Time Autocorrelation Function. The squared time autocorrelation function is used in computation of carrier to noise ratio (CNR). For the proposed channel, it is defined as

$$
\mathbf{R}_{\mu^{2}}(\Delta t)=E\left[|\mu(t)|^{2}|\mu(t-\Delta t)|^{2}\right] .
$$


In Appendix C, it is derived and the final form obtained is

$$
\begin{aligned}
\mathbf{R}_{\mu^{2}}(\Delta t)= & \sigma_{1}^{4} \frac{\left(1+q^{2}\right)^{2}}{q^{4}} \\
& +2\left[\mathbf{R}_{11}^{2}(\Delta t)+\mathbf{R}_{22}^{2}(\Delta t)+2 \mathbf{R}_{12}^{2}(\Delta t)\right],
\end{aligned}
$$

where $\mathbf{R}_{11}$ and $\mathbf{R}_{22}$ are the autocorrelation of in-phase and quadrature components, respectively, where $\mathbf{R}_{12}$ is crosscorrelation between them.

2.3.4. Level Crossing Rate and Average Duration of Fade. The level crossing rate of the process $R(t)$ is obtained by solving the following integral:

$$
N_{R}(r)=\int_{0}^{\infty} \dot{z} p_{R \dot{R}}(r, \dot{z}) d \dot{z},
$$

where $p_{R \dot{R}}$ is the joint PDF of $R(t)$ and its time derivative $\dot{R}(r)$. From [22], LCR for stationary to mobile Hoyt channel is given by

$$
\begin{aligned}
N_{R}(r)= & \frac{r}{(2 \pi)^{3 / 2} \sigma_{1} \sigma_{2}} \\
& \times \int_{0}^{2 \pi} e^{\left[-\left(r^{2} / 2 \sigma_{1}^{2} \sigma_{2}^{2}\right)\left(\sigma_{2}^{2} \cos ^{2}(\theta)+\sigma_{1}^{2} \sin ^{2}(\theta)\right)\right]} \\
& \times \sqrt{\beta_{1} \cos ^{2}(\theta)+\beta_{2} \sin ^{2}(\theta)} d \theta
\end{aligned}
$$

The expression will remain the same for V2V NakagamiHoyt channel. The only thing that will differ here will be the values of $\beta_{1}$ and $\beta_{2}$. These can be found in Appendix B using the relationship $\beta_{i}=-\ddot{\mathbf{R}}_{\mu}(0)$. Also, substituting $\beta_{2}=\beta_{1} / q^{2}$ and $\rho=R / R_{\text {rms }}$, the LCR is obtained as

$$
\begin{aligned}
N_{R}(\rho)= & \frac{\rho \sqrt{\beta_{1}\left(q^{2}+1\right)}}{(2 \pi)^{3 / 2} q \sigma_{1}} \\
& \times \int_{0}^{2 \pi} e^{\left[-\left(\rho^{2}\left(q^{2}+1\right) / 2 q^{2}\right)\left(\cos ^{2}(\theta)+q^{2} \sin ^{2}(\theta)\right)\right]} \\
& \times \sqrt{q^{2} \cos ^{2}(\theta)+\sin ^{2}(\theta)} d \theta
\end{aligned}
$$

For isotropic scattering, from Appendix B, substituting the values of $\beta_{1}=\left(\sqrt{2} \pi \sigma_{1} f_{m 1}\right)^{2}\left(1+a^{2}\right)$, the expression becomes

$$
\begin{aligned}
N_{R}(\rho)= & \frac{\sqrt{\left(1+a^{2}\right)\left(q^{2}+1\right)} f_{m 1} \rho}{2 q \sqrt{\pi}} \\
& \times \int_{0}^{2 \pi} e^{\left[-\left(\rho^{2}\left(q^{2}+1\right) / 2 q^{2}\right)\left(\cos ^{2}(\theta)+q^{2} \sin ^{2}(\theta)\right)\right]} \\
& \times \sqrt{q^{2} \cos ^{2}(\theta)+\sin ^{2}(\theta)} d \theta .
\end{aligned}
$$

It is easy to show that by substituting $a=0$ and $q=1$, the above equation is reduced to the expression for base to mobile Rayleigh LCR given by [40].

The average duration of fade of a signal is defined as average duration of time for which the signal $r$ spends below a specified threshold $R_{0}$. It is given by [41]

$$
\bar{\tau}=\frac{P\left(r<R_{0}\right)}{N_{R}(r)},
$$

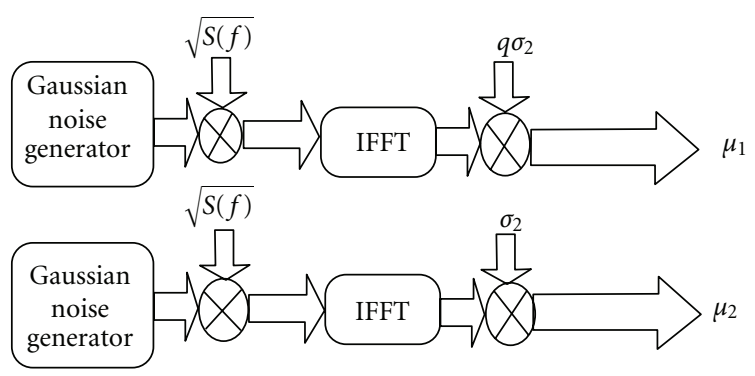

FIGURE 2: Block diagram of IFFT based simulator.

where $P\left(r<R_{0}\right)$ is the cumulative density function obtained by

$$
P\left(r<R_{0}\right)=\int_{0}^{R_{0}} p_{R}(x) d x .
$$

This is obtained by integrating (7). Hence substituting (31) and (33) in (32), ADF can be directly obtained.

\section{Simulation and Results}

The simulator described in this paper uses Smith spectrum method used in [42]. This method is IFFT based and was slightly modified to generate Hoyt Fading signal envelope. This method requires frequency domain generation and processing of random signal followed by inverse Fourier transform to obtain a time domain sequence with the desired properties. The block diagram of the proposed simulator is shown in Figure 2. To implement the simulator, the following steps are performed.

(1) Input number of frequency samples $(N)$ and time samples $(M)$.

(2) Specify maximum doppler frequency of the receiver $f_{m 1}$ in $\mathrm{Hz}$.

(3) Specify the value of parameter $a$ such that maximum Doppler frequency of transmitter $f_{m 2}=a f_{m 1}$.

(4) Specify the value of $q$.

(5) Generate two N/2 samples Gaussian quadrature components of zero mean and unity variance. Generate the remaining $N / 2$ components by conjugating them. This forms the negative frequency components.

(6) Generate $N$ points spectrum $\sqrt{S(f)}$.

(7) The frequency spacing between the adjacent spectral lines is given by $\Delta f=2 f_{m 1}(1+a) / N$.

(8) The time resolution is given by $1 / \Delta f(M-1)$.

(9) Multiply the in-phase and quadrature components by $\sqrt{S(f)}$ and perform the IFFT of the resultant individual. Normalize both the resulting in-phase and quadrature to make their variance unity.

(10) Quadrature component will yield $\mu_{2}$ while in-phase component after multiplying with $q$ will yield $\mu_{1}$. 


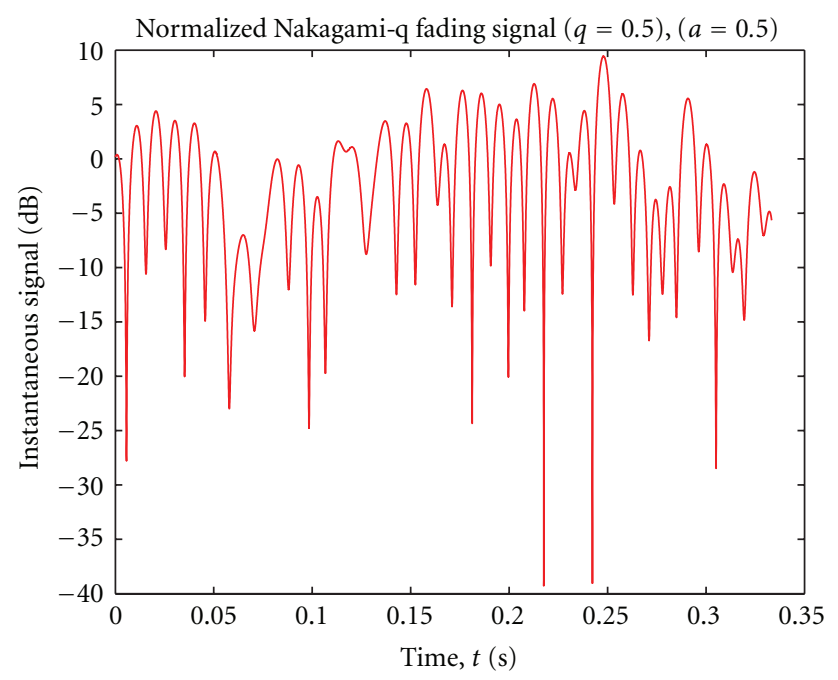

Figure 3: Output of the hoyt simulator.

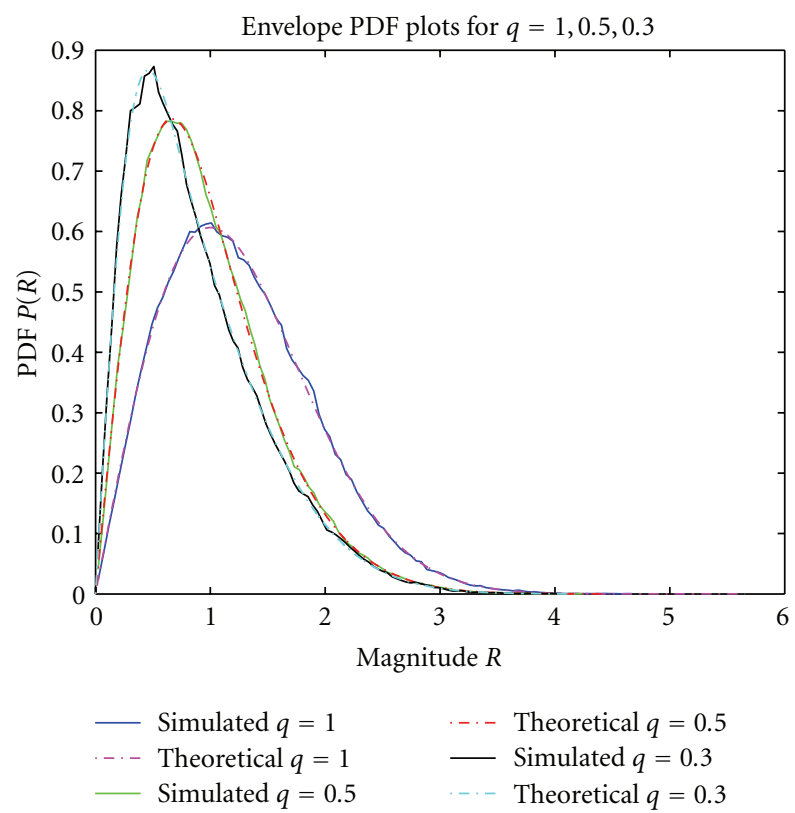

FIGURE 4: Hoyt amplitude PDF plot $q=0.5, a=0.5, k=3$.

(11) The root of the sum of squared envelop of both will generate random variable Nakagami Hoyt distribution for the given value of $q$.

(12) The phase distribution is obtained by using the phase random variable $\tan ^{-1}\left(\mu_{1} / \mu_{2}\right)$.

The simulation was run with the following parameters, carrier frequency $f=900 \mathrm{MHz}$, velocity of receiver $V_{1}=$ $72 \mathrm{~km} / \mathrm{hr}$ which means $f_{m 1}=60 \mathrm{~Hz}$, three different values of $q=1,0.5,0.3$ and three different values of $a=1,0.5,0$. The simulator sample output for $q=0.5, a=0.5$ is shown in Figure 3. The amplitude and phase pdf plots are shown in Figures 4 and 5, respectively. The corresponding theoretical output of (7) and (8), respectively was also plotted for comparison.

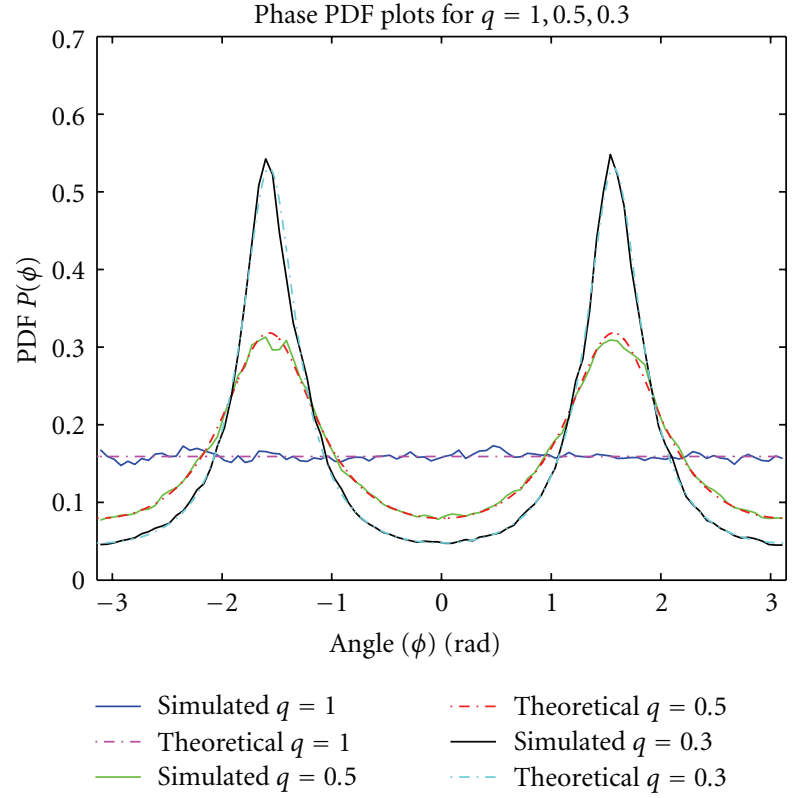

FIGURE 5: Hoyt phase PDF plot $q=0.5, a=0.5, k=3$.

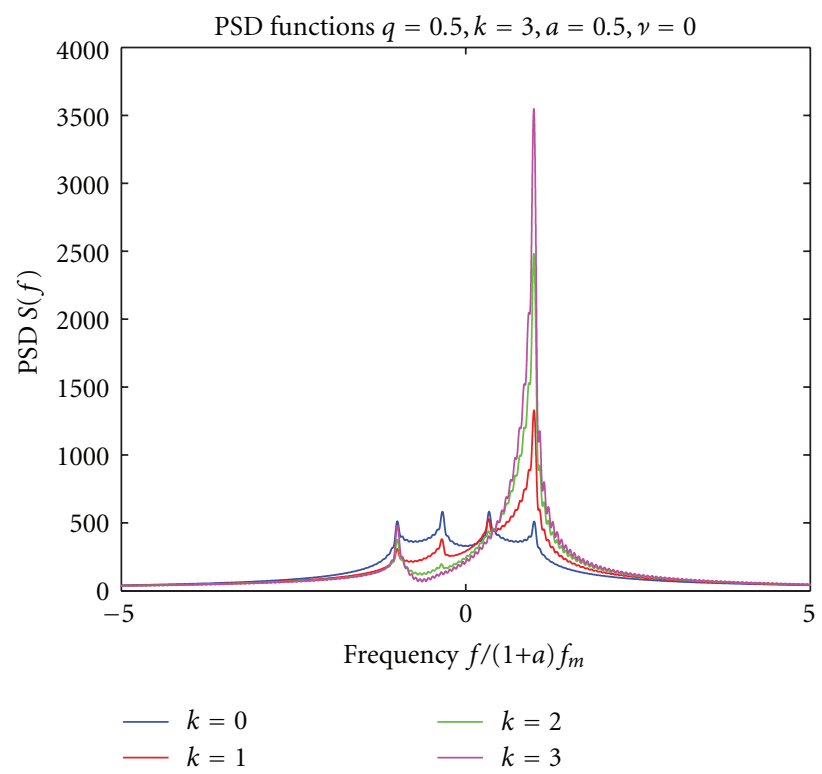

FIgURE 6: PSD plot for $q=0.5$.

The power spectral density plots for $\kappa=(0,1,2,3)$ are shown in Figure 6. The plots for other values of $q$ are the scaled version of this and are not shown here. It has been evident from the plot that $S(f)$ has peaks at $f=$ $\pm\left(f_{m 1}-f_{m 2}\right)$. It has been shown that plot is symmetric for $\kappa=0$ indicating isotropic scattering whereas $\kappa \neq 0$ results in asymmetric PSD (non-isotropic scattering).

The autocorrelation plots for $q=0.5$ and $a=0.5$ with 4 different values of $\kappa=(0,1,2,3)$ are shown in Figure 7 . Plot for $\kappa=0$ indicates $\mathrm{V} 2 \mathrm{~V}$ isotropic scattering. The normalized autocorrelation functions plots have been shown in Figure 8. The plots for $a=1,0.5,0, q=0.5$, and $\kappa=3$ are 


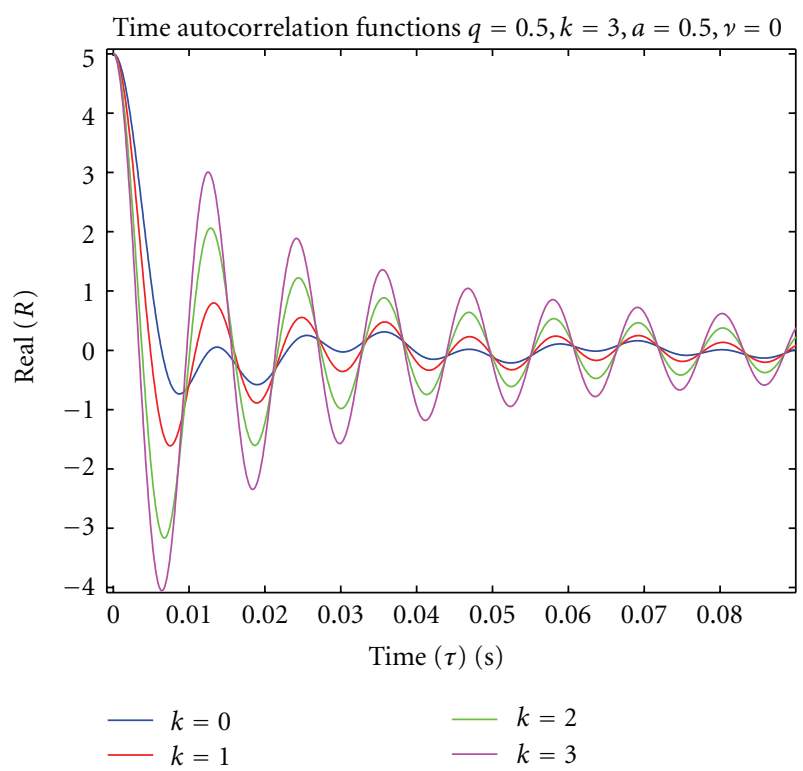

FIgURE 7: Time autocorrelation function of real part of envelope.

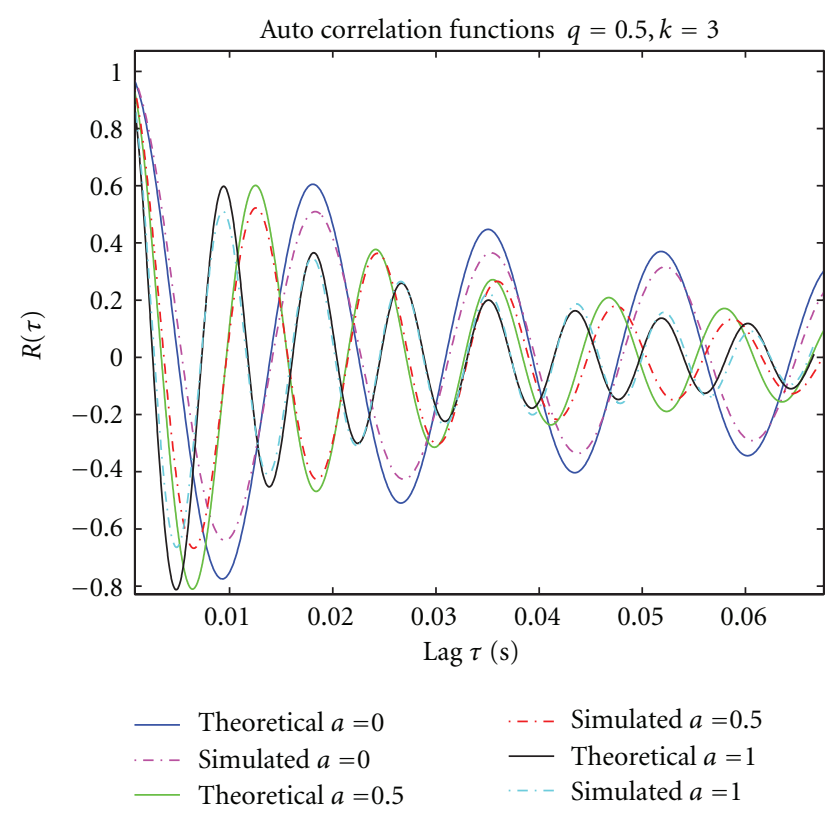

Figure 8: Time Autocorrelation function of Real part of Envelope for $q=0.5, k=3$.

compared with the theoretical expression given in (17). Since the real and imaginary components are Gaussian, it can be found from the plots that the normalized autocorrelation functions are still Bessel but with different shape than the one shown in [15]. Also the plots are function of $\kappa$ and $\nu$.

The normalized squared autocorrelation plots of real part for $q=0.5, \kappa=3, a=0.5$ is shown in Figure 9. The plot is compared with the theoretical derived expression.

The LCR and ADF of envelop for $q=(1,0.5,0.3)$ and $\kappa=3$ are plotted in Figures 10, 11, 12, 13, 14, and 15 for three different values of $a=(1,0.5,0)$. The curves are matched with their theoretical expressions given by (31) and (32). $q=$ 1 shows the Rayleigh envelop whereas $a=0$ indicates base to mobile communication plots for LCR and ADF.

The mean square error (MSE) of the time autocorrelation function is given by

$$
\mathrm{MSE}=E\left[\left(\mathbf{R}_{\mu} \Delta t-\widehat{\mathbf{R}_{\mu}} \Delta t\right)^{2}\right],
$$

where $\mathbf{R}_{u} \Delta t$ and $\widehat{\mathbf{R}_{u}} \Delta t$ are the theoretical and estimated autocorrelation functions, respectively. Figure 16 shows the mean square error of time autocorrelation function as a function of number of frequency sample points $N$. The figure is obtained for $q=0.5, a=0.5, \kappa=3$, and varying $N$ in power of 2 in the range 2048-32768. It is evident from the curve that the MSE reduces when the number of sample points is increased. Hence, more accurate simulator is obtained at the cost of increasing the complexity of simulator.

\section{Conclusion}

The second order statistical properties for vehicle to vehicle Nakagami-Hoyt channels, under the non-isotropic scattering conditions at both the transmitter and receiver, have been developed. These include expressions for space time correlation function, power spectral density, squared time autocorrelation, level crossing rates, and average duration of fade. The Nakagami-Hoyt V2V simulator has also been developed to verify the above mentioned theoretical expressions. It has been found that the theoretical results match closely with the simulated data verifying the validity of the model.

\section{Appendices}

\section{A. Proof of the Spatial Time Correlation Function}

The spatial time correlation function is given by

$$
\mathbf{R}\left(x_{1}, x_{2}, t_{1}, t_{2}\right)=\frac{1}{2}\left\langle\mu_{x_{2}}\left(t_{2}\right) \mu_{x_{1}}^{*}\left(t_{1}\right)\right\rangle,
$$

where $t_{2}=t_{1}+\Delta t$. Using [14] as a reference

$$
\begin{aligned}
\mu_{x_{2}}\left(t_{2}\right)= & \mu_{x_{2}}\left(t_{1}+\Delta t\right) \\
= & \sum_{i=1}^{N} r_{i} \cos \left[\left(\omega_{1 i}+\omega_{2 i}\right)\left(t_{1}+\Delta t\right)+\phi_{i}+\psi_{i}\right] \\
& +j \frac{1}{q} \sum_{i=1}^{N} r_{i} \sin \left[\left(\omega_{1 i}+\omega_{2 i}\right)\left(t_{1}+\Delta t\right)+\phi_{i}+\psi_{i}\right],
\end{aligned}
$$




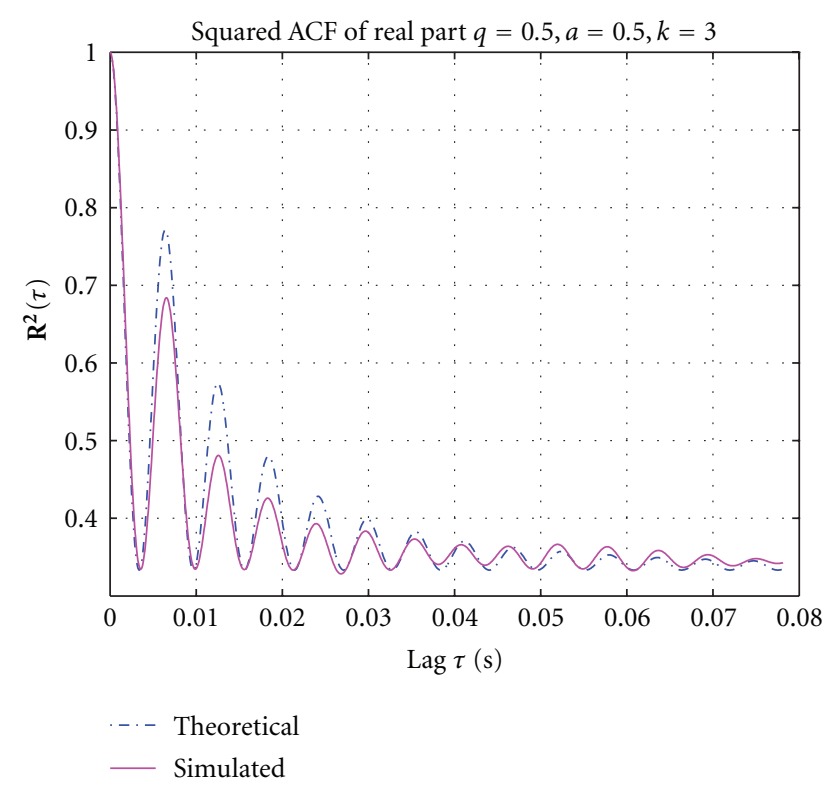

FIgURE 9: Squared Autocorrelation function of Real part for $q=$ $0.5, k=3$.

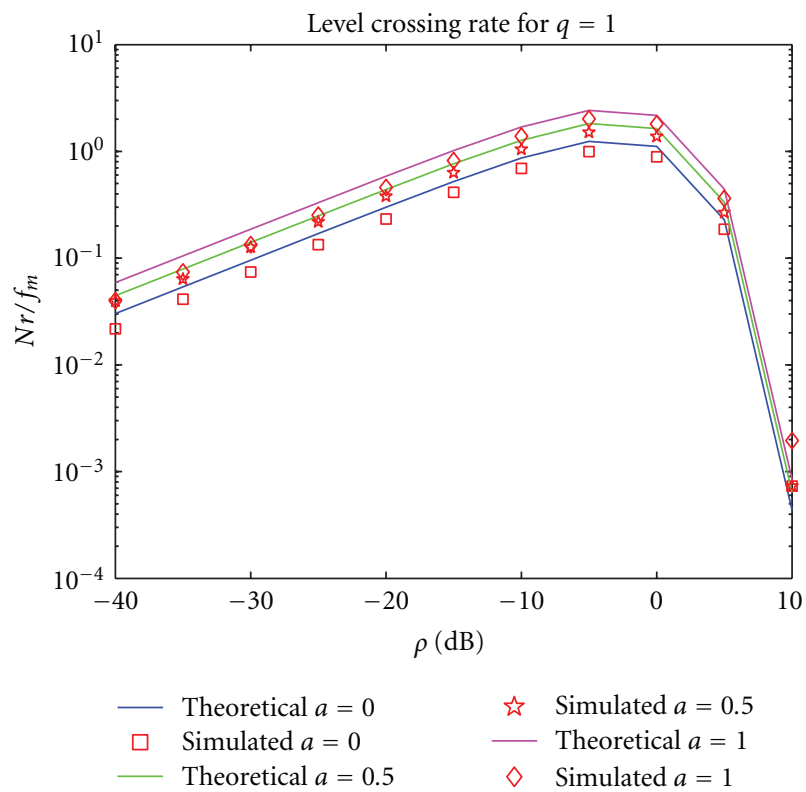

Figure 10: Level crossing rates for $q=1, k=3$.

where $\psi_{i}=(2 \pi / \lambda) \Delta x \cos \alpha_{1 i}, \omega_{l i}=2 \pi f_{m l} \cos \alpha_{l i}, l=1,2, \phi_{i}$ is the uniformly distributed phase, and $\alpha_{1 i}$ is the AOA of the $i$ th component. Also,

$$
\begin{aligned}
\mu_{x_{1}}\left(t_{1}\right)= & \sum_{i=1}^{N} r_{i} \cos \left[\left(\omega_{1 i}+\omega_{2 i}\right) t_{1}+\phi_{i}\right] \\
& +j \frac{1}{q} \sum_{i=1}^{N} r_{i} \sin \left[\left(\omega_{1 i}+\omega_{2 i}\right) t_{1}+\phi_{i}\right] .
\end{aligned}
$$

Therefore, we obtain,

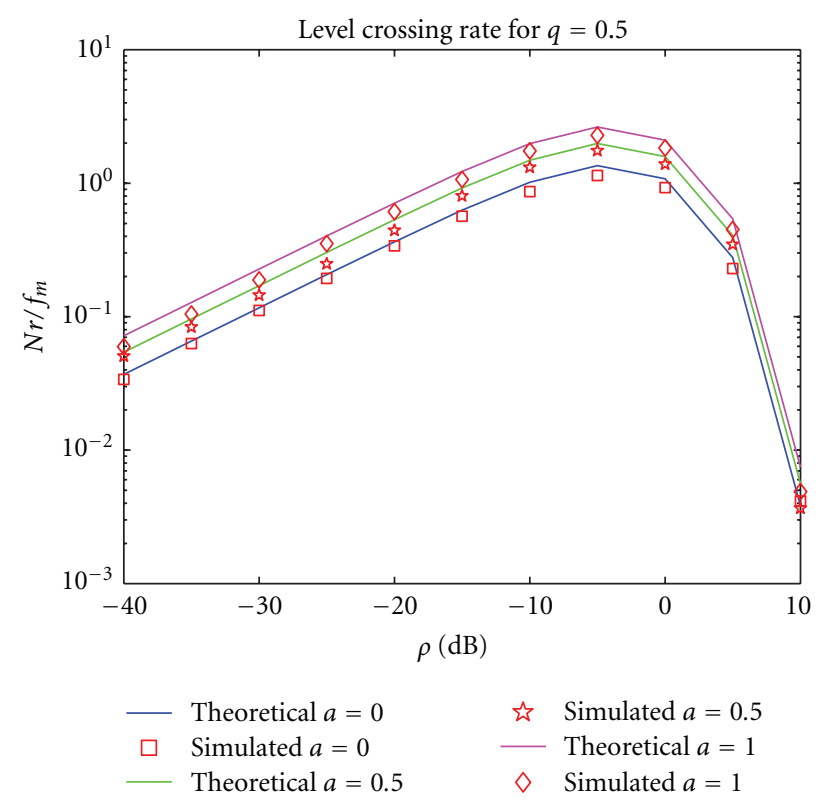

FIGURE 11: Level crossing rates for $q=0.5, k=3$.

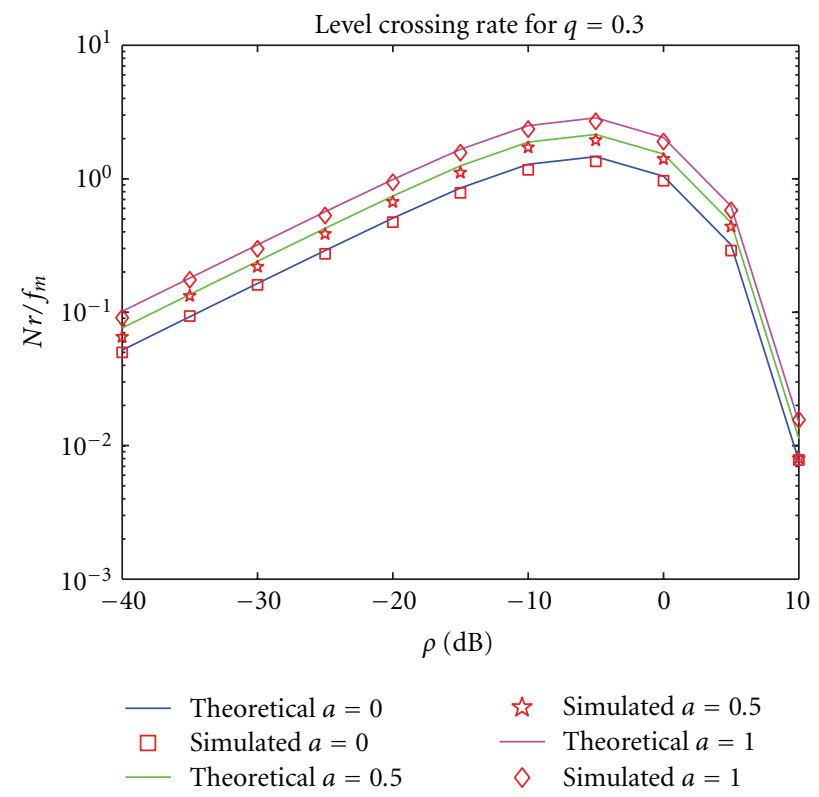

FIGURE 12: Level crossing rates for $q=0.3, k=3$.

$$
\begin{aligned}
& \mathbf{R}\left(x_{1}, x_{2}, t_{1}, t_{2}\right) \\
& =\frac{1}{2}\left\langle\sum_{i=1}^{N} r_{i} r_{j} \sum_{j=1}^{N} r_{i} r_{j}\right. \\
& \times\left\{\begin{array}{l}
\cos \left[\left(\omega_{1 i}+\omega_{2 i}\right)\left(t_{1}+\Delta t\right)+\phi_{i}+\psi_{i}\right] \\
\times \cos \left[\left(\omega_{1 j}+\omega_{2 j}\right) t_{1}+\phi_{j}\right]
\end{array}\right.
\end{aligned}
$$




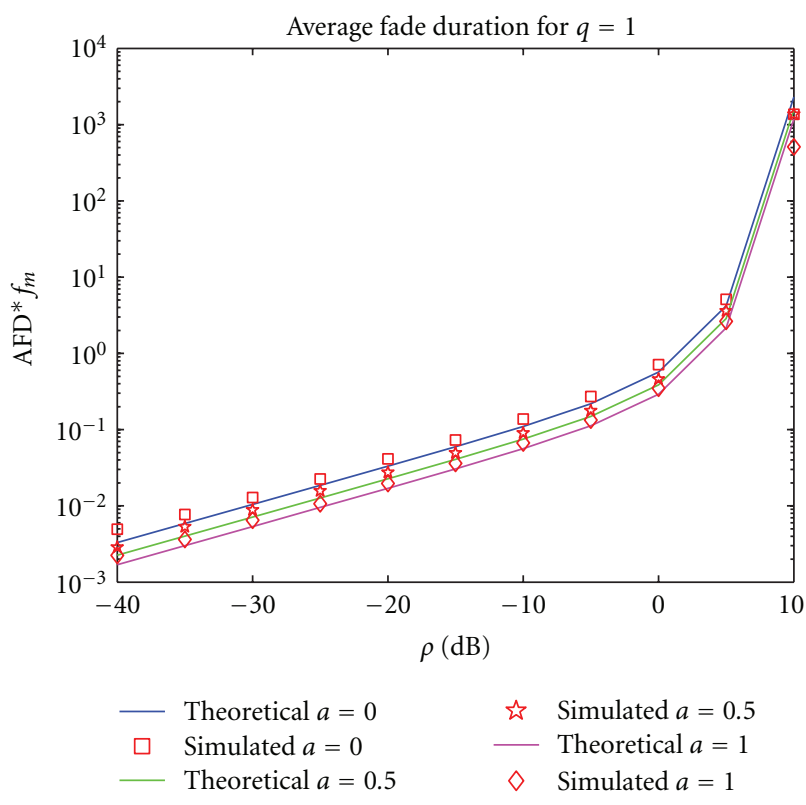

Figure 13: Average duration of fade for $q=1, k=3$.

$$
\begin{aligned}
& +\frac{1}{q^{2}} \sin \left[\left(\omega_{1 i}+\omega_{2 i}\right)\left(t_{1}+\Delta t\right)+\phi_{i}+\psi_{i}\right] \\
& \times \sin \left[\left(\omega_{1 j}+\omega_{2 j}\right) t_{1}+\phi_{j}\right] \\
& +j \frac{1}{q} \sin \left[\left(\omega_{1 i}+\omega_{2 i}\right)\left(t_{1}+\Delta t\right)+\phi_{i}+\psi_{i}\right] \\
& \times \cos \left[\left(\omega_{1 j}+\omega_{2 j}\right) t_{1}+\phi_{j}\right]
\end{aligned}
$$

$$
\begin{aligned}
& -j \frac{1}{q} \cos \left[\left(\omega_{1 i}+\omega_{2 i}\right)\left(t_{1}+\Delta t\right)+\phi_{i}+\psi_{i}\right] \\
& \left.\left.\times \sin \left[\left(\omega_{1 j}+\omega_{2 j}\right) t_{1}+\phi_{j}\right]\right\}\right\rangle .
\end{aligned}
$$

Assuming $\phi_{i}, \alpha_{1 i}, \alpha_{2 i}$, and $r_{i}$ are mutually independent. Also, assume that $\alpha_{1 i}$ and $\alpha_{2 i}$ have same distributions for all $i$. Since $\phi_{i}$ is assumed to be uniformly distributed, therefore $E\left[e^{j\left(\phi_{i}-\phi_{j}\right)}\right]=0$ for all $i \neq j$. Hence for $i=j, E\left[e^{j\left(\phi_{i}-\phi_{j}\right)}\right]=1$ and we get

$$
\begin{aligned}
\mathbf{R}(\Delta x, \Delta t)=\frac{1}{2}\langle & \sum_{i=1}^{N} r_{i}^{2} \\
& \times\left\{\left(1+\frac{1}{q^{2}}\right) \cos \left[\left(\omega_{1 i}+\omega_{2 i}\right) \Delta t+\psi_{i}\right]\right. \\
& \left.\left.+j \frac{2}{q} \sin \left[\left(\omega_{1 i}+\omega_{2 i}\right) \Delta t+\psi_{i}\right]\right\}\right\rangle .
\end{aligned}
$$

Also, assuming

$$
\sigma_{1}^{2}=\frac{1}{2}\left\langle\sum_{i=1}^{N} r_{i}^{2}\right\rangle
$$

Using the Euler identities for sine and cosine and the formula given in [29] for Von Mises distribution of $\alpha_{i}$, we get

$$
\begin{aligned}
E\left[e^{j w_{1} \Delta t+(2 \pi / \lambda) \Delta x \cos \alpha_{1}}\right] & =\frac{1}{2 \pi I_{0}\left(\kappa_{1}\right)} \int_{-\pi}^{\pi} e^{\kappa_{1} \cos \left(\alpha_{1}-\nu_{1}\right)} e^{j\left(w_{1} \Delta t+(2 \pi / \lambda) \Delta x \cos \alpha_{1}\right)} d \alpha_{1} \\
& =\frac{I_{0}\left(\sqrt{\kappa_{1}^{2}+\left(\omega_{1} \Delta t+(2 \pi / \lambda) \Delta x\right)^{2}+j \kappa_{1}\left(\omega_{1} \Delta t+(2 \pi / \lambda) \Delta x\right) \cos \nu_{1}}\right)}{I_{0}\left(\kappa_{1}\right)} \\
E\left[e^{j w_{2} \Delta t}\right] & =\frac{1}{2 \pi I_{0}\left(\kappa_{2}\right)} \int_{-\pi}^{\pi} e^{\kappa_{2} \cos \left(\alpha_{2}-\nu_{2}\right)+j\left(w_{2} \Delta t\right)} d \alpha_{2} \\
& =\frac{I_{0}\left(\sqrt{\kappa_{2}^{2}+\omega_{2}^{2} \Delta t^{2}+j 2 \kappa_{2} \omega_{2} \Delta t \cos \nu_{2}}\right)}{I_{0}\left(\kappa_{2}\right)} .
\end{aligned}
$$

Hence, after substituting $\omega_{i}=K V_{i}$ and further simplification, we obtain

$$
\mathbf{R}(\Delta x, \Delta t)=\sum_{n=0}^{1} \frac{(1+q \cos (n \pi))^{2}}{4 q^{2}} \sigma_{1}^{2} \prod_{i=1}^{2} \frac{I_{0}\left(\sqrt{\kappa_{i}^{2}-K^{2} M_{i}^{2}+j 2 \kappa_{i} K M_{i} \cos \nu_{i} \cos (n \pi)}\right)}{I_{0}\left(\kappa_{i}\right)}
$$

where

$$
M_{i}(\Delta x, \Delta t)=V_{i} \Delta t+(2-i) \Delta x
$$

\section{B. Values of Beta}

The values of $\beta_{i}$ are calculated from

$$
\beta_{i}=-\ddot{\mathbf{R}}_{i i}(0),
$$




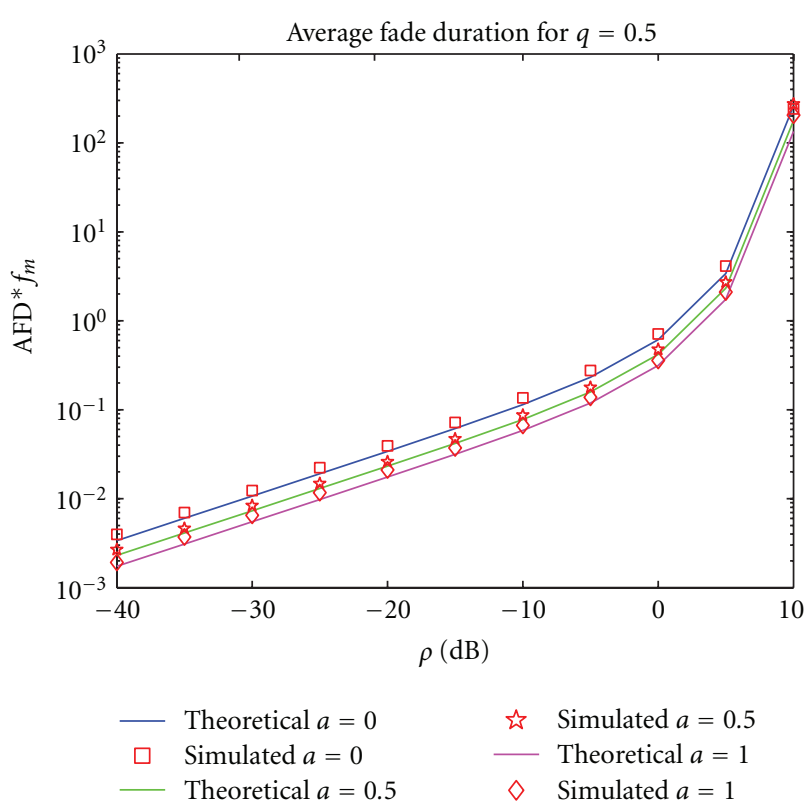

Figure 14: Average duration of fade for $q=0.5, k=3$.

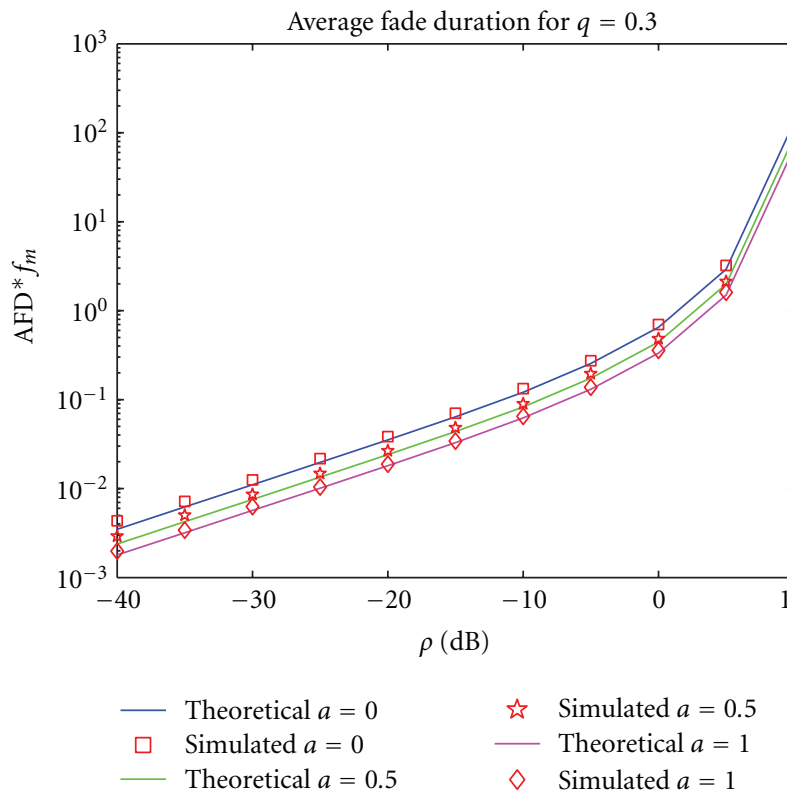

FIGURE 15: Average duration of fade for $q=0.3, k=3$.

where

$$
\begin{aligned}
& \mathbf{R}_{i i}(\Delta t)=E\left[\mu_{i}(t+\Delta t) \mu_{i}(t)\right] \quad i=1,2 \\
& \mathbf{R}_{11}(\Delta t) \\
& =\sigma_{1}^{2} \sum_{n=0}^{1} \prod_{i=1}^{2} \frac{I_{0}\left(\sqrt{\kappa_{i}^{2}-\left(K V_{i} \Delta t\right)^{2}+j 2 \kappa_{i} K V_{i} \Delta t \cos \nu_{i} \cos (n \pi)}\right)}{I_{0}\left(\kappa_{i}\right)} .
\end{aligned}
$$

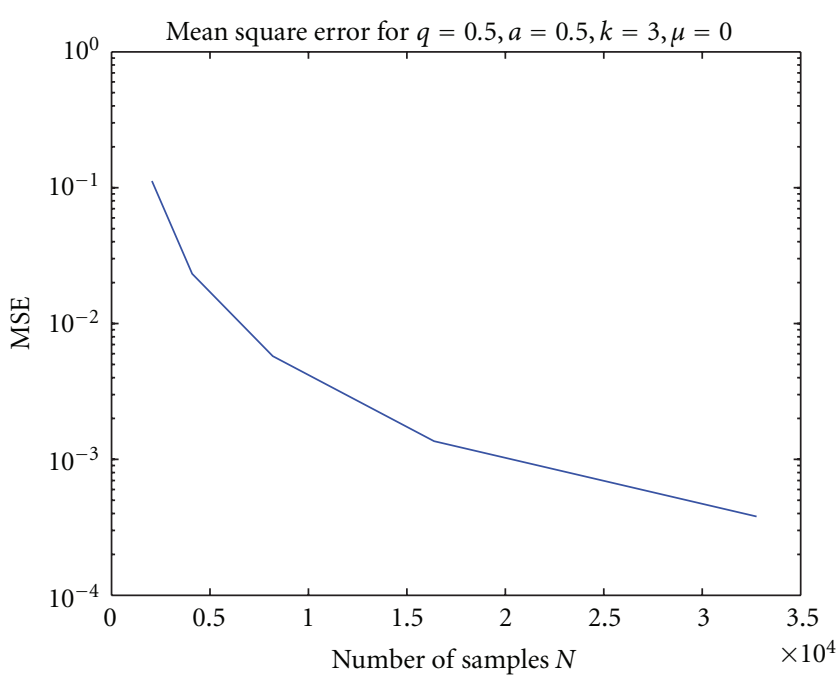

Figure 16: Mean square error of autocorrelation function $q=$ $0.5, a=0.5, k=3$.

Differentiating twice and substituting $\Delta t=0$ yields

$$
\begin{aligned}
\beta_{1}=-\mathbf{R}_{11}(0) & \\
=\sigma_{1}^{2} K^{2} V_{1}^{2}[ & 2 a^{2} \cos \nu_{1} \cos \nu_{2} \frac{I_{1}\left(\kappa_{1}\right) I_{1}\left(\kappa_{2}\right)}{I_{0}\left(\kappa_{1}\right) I_{0}\left(\kappa_{2}\right)} \\
& \quad-\frac{I_{1}\left(\kappa_{1}\right) \cos 2 \nu_{1}}{\kappa_{1} I_{0}\left(\kappa_{1}\right)}-a^{2} \frac{I_{1}\left(\kappa_{2}\right) \cos 2 \nu_{2}}{\kappa_{2} I_{0}\left(\kappa_{2}\right)} \\
& \left.+\cos ^{2} \nu_{1}+a^{2} \cos ^{2} \nu_{2}\right] .
\end{aligned}
$$

Provided $\kappa_{1}, \kappa_{2} \neq 0$.

Similarly, we get $\beta_{2}=\beta_{1} / q^{2}$.

For the case of isotropic scattering $\left(\kappa_{1}=\kappa_{2}=\nu_{1}=\right.$ $\left.\nu_{2}=0\right)$, differentiating (17) with respect to $\Delta t$ twice and substituting $\Delta t=0$, we get

$$
\beta_{1}=-\ddot{\mathbf{R}}_{11}(0)=\left(\sqrt{2} \pi \sigma_{1} f_{m 1}\right)^{2}\left(1+a^{2}\right) .
$$

\section{Proof of the Squared Time Autocorrelation Function}

The squared time autocorrelation function is given by (26) and can be written as

$$
\begin{aligned}
\mathbf{R}_{\mu^{2}}(\Delta t)= & E\left[\mu_{1}^{2}(t) \mu_{1}^{2}(t-\Delta t)\right]+E\left[\mu_{2}^{2}(t) \mu_{2}^{2}(t-\Delta t)\right] \\
& +E\left[\mu_{1}^{2}(t) \mu_{2}^{2}(t-\Delta t)\right]+E\left[\mu_{2}^{2}(t) \mu_{1}^{2}(t-\Delta t)\right]
\end{aligned}
$$

For zero mean Gaussian random variable we have from Chapter 6 [43],

$$
\begin{aligned}
E\left[\mu_{1}^{2}(t) \mu_{1}^{2}(t-\Delta t)\right]= & E\left[\mu_{1}^{2}(t)\right] E\left[\mu_{1}^{2}(t-\Delta t)\right] \\
& +2 E^{2}\left[\mu_{1}(t) \mu_{1}(t-\Delta t)\right] \\
= & \sigma_{1}^{4}+2 \mathbf{R}_{11}^{2}(\Delta t) .
\end{aligned}
$$


Similarly,

$$
\begin{gathered}
E\left[\mu_{2}^{2}(t) \mu_{2}^{2}(t-\Delta t)\right]=\sigma_{2}^{4}+2 \mathbf{R}_{22}^{2}(\Delta t), \\
E\left[\mu_{1}^{2}(t) \mu_{2}^{2}(t-\Delta t)\right]=\sigma_{1}^{2} \sigma_{2}^{2}+2 \mathbf{R}_{12}^{2}(\Delta t) .
\end{gathered}
$$

Hence substituting in (26) yields

$$
\mathbf{R}_{\mu^{2}}(\Delta t)=\sigma_{1}^{4} \frac{\left(1+q^{2}\right)^{2}}{q^{4}}+2\left[\mathbf{R}_{11}^{2}(\Delta t)+\mathbf{R}_{22}^{2}(\Delta t)+2 \mathbf{R}_{12}^{2}(\Delta t)\right],
$$

where

$$
\mathbf{R}_{12}(\Delta t)=\frac{1}{q} \sigma_{1}^{2}\left[\sum_{n=0}^{1} \cos (n \pi) \prod_{i=1}^{2} \frac{I_{0}\left(\sqrt{\kappa_{i}^{2}-\left(K V_{i} \Delta t\right)^{2}+j 2 \kappa_{i} K V_{i} \Delta t \cos \nu_{i} \cos (n \pi)}\right)}{I_{0}\left(\kappa_{i}\right)}\right] .
$$

\section{Acknowledgments}

The authors would like to acknowledge the support provided by King Abdulaziz City for Science and Technology (KACST) through the Science and Technology Unit at King Fahd University of Petroleum and Minerals (KFUPM) for funding this work through Project no. NSTP08-ELEC42-4 as part of the National Science, Technology and Innovation Plan (NSTIP).

\section{References}

[1] S. O. Rice, "Statistical properties of a sine wave plus random noise," Bell System Technical Journal, vol. 27, pp. 109-157, 1948.

[2] R. S. Hoyt, "Probability functions for the modulus and angle of the normal complex variate," Bell System Technical Journal, vol. 26, pp. 318-359, 1947.

[3] M. Nakagami, "The m-distribution-a general formula of intensity distribution of rapid fading," in Statistical Methods in Radio Wave Propagation, W. G. Hoffman, Ed., pp. 3-36, Permagon Press, Oxford, UK, 1960.

[4] M. A. Taneda, J. Takada, and K. Araki, "A new approach to fading: weibull model," in Proceedings of the IEEE International Symposium on Personal, Indoor, and Mobile Radio Communications, pp. 711-715, Osaka, Japan, September 1999.

[5] G. L. Turin, F. D. Clapp, T. L. Johnston et al., "A statistical model of urban multipath propagation," in Proceedings of the IEEE Transactions on Vehicular Technology, vol. VT-21, p. 19, February 1972.

[6] F. Hansen and F. I. Meno, "Mobile fading-Rayleigh and lognormal superimposed," IEEE Transactions on Vehicular Technology, vol. VT-26, no. 4, pp. 332-335, 1977.

[7] H. Suzuki, "Statistical model for urban radio propagation," IEEE Transactions on Communications, vol. 25, no. 7, pp. 673680, 1977.

[8] F. Vatalaro and G. E. Corazza, "Probability of error and outage in a rice-lognormal channel for terrestrial and satellite personal communications," IEEE Transactions on Communications, vol. 44, no. 8, pp. 921-924, 1996.

[9] Safe and Comfortable Driving Based Upon Inter-Vehicle Communication, 2001, http://www.cartalk2000.net/.

[10] Car2Car Communication Consortium, 2005, http://www.carto-car.org/.

[11] Project MobiVip, 2005, http://www-sop.inria.fr/mobivip/.

[12] FleetNet project Internet on the road, 2000, http://www.neclab .eu/Projects/fleetnet.htm.
[13] C. F. Mecklenbraüker, A. F. Molisch, J. Karedal et al., "Vehicular channel characterization and its implications for wireless system design and performance," Proceedings of the IEEE, vol. 99, no. 7, pp. 1189-1212, 2011.

[14] A. S. Akki and F. Haber, "Statistical model of mobile-to-mobile land communication channel," IEEE Transactions on Vehicular Technology, vol. VT-35, no. 1, pp. 2-7, 1986.

[15] A. S. Akki, "Statistical properties of mobile-to-mobile land communication channels," IEEE Transactions on Vehicular Technology, vol. 43, no. 4, pp. 826-831, 1994.

[16] R. Wang and D. Cox, "Channel modeling for ad hoc mobile wireless networks," in Proceedings of the IEEE Vehicular Technology Conference, vol. 1, pp. 21-25, Birmingham, UK, May 2002.

[17] C. S. Patel, G. L. Stuber, and T. G. Pratt, "Simulation of Rayleigh faded mobile-to-miobile comimunication channels," in Proceedings of the 58th IEEE Vehicular Technology Conference (VTC '03), pp. 163-167, Orlando, Fla, USA, October 2003.

[18] B. O. Hogstad, M. Pätzold, N. Youssef, and D. Kim, "A MIMO mobile-to-mobile channel model: part II-the simulation model," in Proceedings of the IEEE 16th International Symposium on Personal, Indoor and Mobile Radio Communications (PIMRC '05), pp. 562-567, Berlin, Germany, September 2005.

[19] A. Petrolino, J. Gomes, and G. Tavares, "A mobile-to-mobile fading channel simulator based on an orthogonal expansion," in Proceedings of the IEEE 67th Vehicular Technology Conference-Spring (VTC '08), pp. 366-370, Singapore, May 2008.

[20] K. C. Borries and D. D. Stancil, "Efficient simulation of mobile-to-mobile rayleigh fading using Gaussian quadrature," in Proceedings of the IEEE 65th Vehicular Technology Conference (VTC '07), pp. 534-538, Dublin, Ireland, April 2007.

[21] A. G. Zajić and G. L. Stüber, "A new simulation model for mobile-to-mobile rayleigh fading channels," in Proceedings of the IEEE Wireless Communications and Networking Conference (WCNC '06), pp. 1266-1270, Las Vegas, Nev, USA, April 2006.

[22] N. Youssef, C. X. Wang, and M. Pätzold, "A study on the second order statistics of Nakagami-Hoyt mobile fading channels," IEEE Transactions on Vehicular Technology, vol. 54, no. 4, pp. 1259-1265, 2005.

[23] N. Hajri, N. Youssef, and M. Pätzold, "A study on the statistical properties of double Hoyt fading channels," in Proceedings of the 6th International Symposium on Wireless Communication Systems (ISWCS '09), pp. 201-205, Tuscany, Italy, September 2009.

[24] A. Abdi, J. A. Barger, and M. Kaveh, "A parametric model for the distribution of the angle of arrival and the associated correlation function and power spectrum at the mobile 
station," IEEE Transactions on Vehicular Technology, vol. 51, no. 3, pp. 425-434, 2002.

[25] M. D. Austin and G. L. Stuber, "Velocity adaptive handoff algorithms for microcellular systems," IEEE Transactions on Vehicular Technology, vol. 43, no. 3, pp. 549-561, 1994.

[26] J. Salz and J. H. Winters, "Effect of fading correlation on adaptive arrays in digital mobile radio," IEEE Transactions on Vehicular Technology, vol. 43, no. 4, pp. 1049-1057, 1994.

[27] X. Cheng, C. X. Wang, D. I. Laurenson, and A. V. Vasilakos, "Second order statistics of non-isotropic mobile-to-mobile Ricean fading channels," in Proceedings of the IEEE International Conference on Communications (ICC '09), Dresden, Germany, June 2009.

[28] A. Fayziyev, M. Pätzold, and N. Youssef, "On the autocorrelation function of rice processes for unsymmetrical Doppler power spectral densities," in Proceedings of the International Conference on Advanced Technologies for Communications (ATC '10), pp. 118-123, Ho Chi Minh City, Vietnam, October 2010.

[29] Y. R. Zheng, "A non-isotropic model for mobile-to-mobile fading channel simulations," in Proceedings of the Military Communications Conference (MILCOM'06), Washington, DC, USA, October 2006.

[30] M. I. Akram and A. U. H. Sheikh, "On the second order statistical properties of Nakagami Hoyt mobile to mobile fading channel," in Proceedings of the Australasian Telecommunication Networks and Applications Conference (ATNAC '011), pp. 1-5, November 2011.

[31] A. K. Papazafeiropoulos and S. A. Kotsopoulos, "An extended generalized rice model for wireless communications," IEEE Transactions on Vehicular Technology, vol. 59, no. 5, pp. 26042609, 2010.

[32] A. F. Molisch, F. Tufvesson, J. Karedal, and C. F. Mecklenbräuker, "A survey on vehicle-to-vehicle propagation channels," IEEE Wireless Communications, vol. 16, no. 6, pp. 12-22, 2009.

[33] D. W. Matolak, I. Sen, and W. Xiong, "Channel modeling for V2V communications," in Proceedings of the 3rd Annual International Conference on Mobile and Ubiquitous Systems, MobiQuitous, pp. 1-7, San Jose, Calif, USA, July 2006.

[34] D. W. Matolak, I. Sen, W. Xiong, and N. T. Yaskoff, " 5 GHZ wireless channel characterization for vehicle to vehicle communications," in Proceedings of the Military Communications Conference (MILCOM '05), vol. 5, pp. 3016-3022, Atlantic City, NJ, USA, October 2005.

[35] K. Otani and H. Omori, "Distribution of burst error lengths in rayleigh fading radio channels," Electronics Letters, vol. 16, no. 23, pp. 889-891, 1980.

[36] K. Otani, K. Daikoku, and H. Omori, "Burst error performance encountered in digital land mobile radio channel," IEEE Transactions on Vehicular Technology, vol. VT-30, no. 4, pp. 156-160, 1981.

[37] H. S. Wang and N. Moayeri, "Finite-state Markov channela useful model for radio communication channels," IEEE Transactions on Vehicular Technology, vol. 44, no. 1, pp. 163171, 1995.

[38] M. Schwartz, W. R. Bennet, and S. Stein, Communication Systems and Techniques, McGraw-Hill, New York, NY, USA, 1966.

[39] I. S. Gradshteyn and I. M. Ryzhik, Tables of Integrals, Series and Products, Academic Press, New York, NY, USA, 1994.

[40] W. C. Jakes, Microwave Mobile Communications, IEEE Press, Piscataway, NJ, USA, 2nd edition, 1993.
[41] S. O. Rice, "Mathematical analysis of random noise," Bell System Technical Journal, vol. 23, pp. 282-332, 1945.

[42] T. S. Rappaport, Wireless Communications: Principle and Practice, Printice Hall Communications Engineering and Emerging Techgnologies series, 2nd edition, 1996.

[43] A. Papoulis and S. U. Pillai, Probability, Random Variables and Stochastic Processes, McGrae-Hill Higher Education, 4th edition, 1996. 

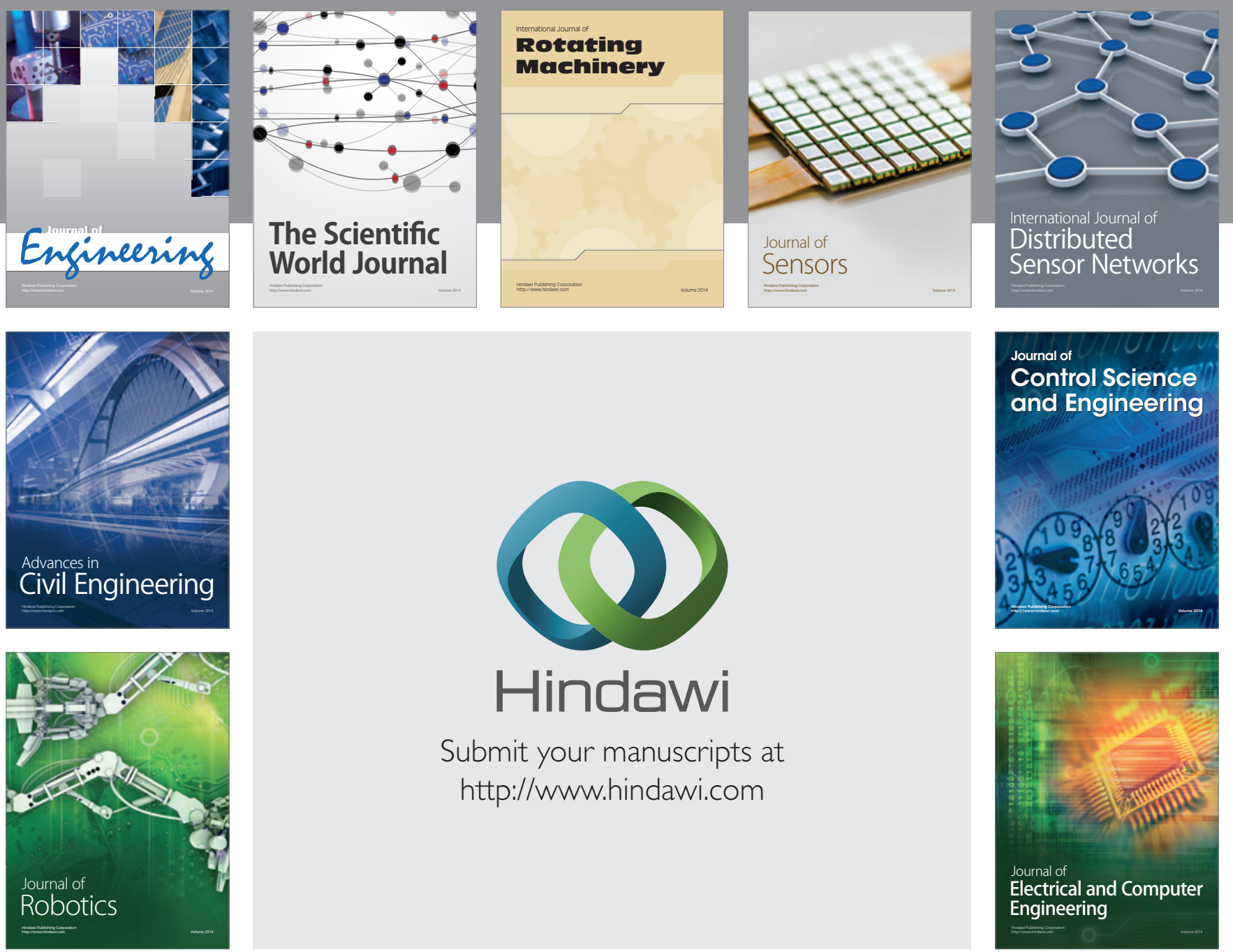

Submit your manuscripts at

http://www.hindawi.com
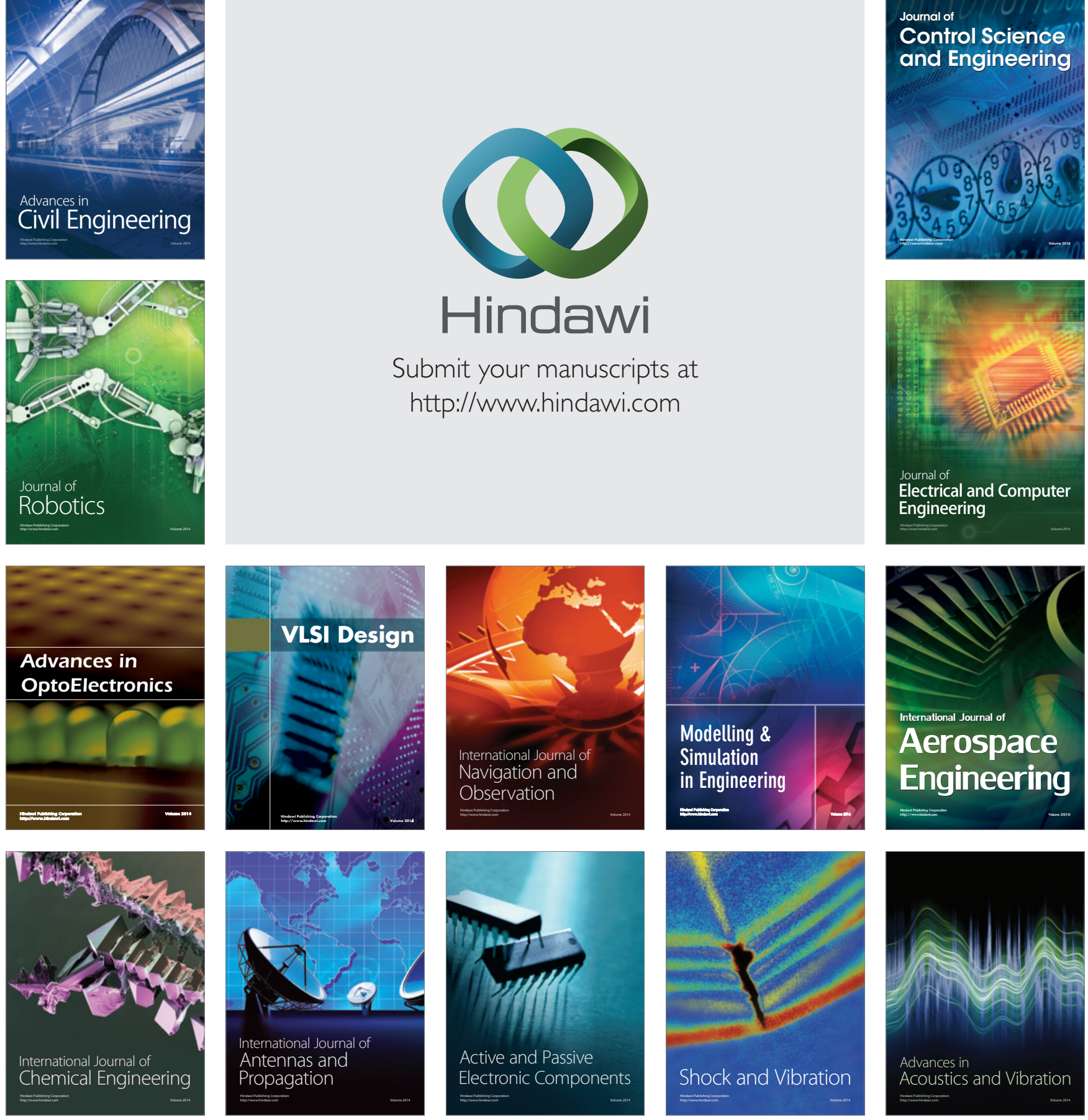